\title{
Benefits and Risks of Iron Supplementation in Anemic Neonatal Pigs
}

\author{
Paweł Lipiński, ${ }^{*}$ Rafał R. Starzyński, ${ }^{*}$ \\ François Canonne-Hergaux, ${ }^{\dagger}$ Barbara Tudek, ${ }^{\ddagger \S}$ \\ Ryszard Oliński, " Paweł Kowalczyk, \\ Tomasz Dziaman, ${ }^{\text {"I }}$ Olivier Thibaudeau, ${ }^{* *}$ \\ Mikołaj A. Gralak, ${ }^{\dagger \dagger}$ Ewa Smuda, ${ }^{*}$ \\ Jarosław Woliński, ${ }^{\ddagger \ddagger}$ Agnieszka Usińska, ${ }^{*}$ \\ and Romuald Zabielski ${ }^{\dagger \dagger}$
}

\begin{abstract}
From the Department of Molecular Biology," Institute of Genetics and Animal Breeding, Polish Academy of Sciences, Jastrzebiec, Poland; the Institut de Chimie des Substances Naturelles, ${ }^{\dagger}$ CNRS, Gif-sur-Yvette, France; the Institute of Biochemistry and Biophysics, ${ }^{\ddagger}$ Polish Academy of Sciences, Warsaw, Poland; the Institute of Genetics and Biotechnology, ${ }^{\S}$ and the Interdisciplinary Centre for Mathematical and Computational Modelling," Warsaw University, Warsaw Poland; the Nicolaus Copernicus University, " Bydgoszcz, Poland; the Institut Claude Bernard-IFR2,** Faculté de Médecine Xavier Bichat, Paris, France; the Department of Physiological Sciences, ${ }^{+\dagger}$ Warsaw University of Life Sciences, Poland; and the Kielanowsi Institute of Animal Physiology and Nutrition, ${ }^{\ddagger \ddagger}$ Jabtonna, Poland.
\end{abstract}

Iron deficiency is a common health problem. The most severe consequence of this disorder is iron deficiency anemia (IDA), which is considered the most common nutritional deficiency worldwide. Newborn piglets are an ideal model to explore the multifaceted etiology of IDA in mammals, as IDA is the most prevalent deficiency disorder throughout the early postnatal period in this species and frequently develops into a critical illness. Here, we report the very low expression of duodenal iron transporters in pigs during the first days of life. We postulate that this low expression level is why the iron demands of the piglet body are not met by iron absorption during this period. Interestingly, we found that a low level of duodenal divalent metal transporter 1 and ferroportin, two iron transporters located on the apical and basolateral membrane of duodenal absorptive enterocytes, respectively, correlates with abnormally high expression of hepcidin, despite the poor hepatic and overall iron status of these animals. Parenteral iron supplementation by a unique intramuscular administration of large amounts of iron dextran is current practice for the treatment of IDA in piglets. However, the poten- tial toxicity of such supplemental iron implies the necessity for caution when applying this treatment. Here we demonstrate that a modified strategy for iron supplementation of newborn piglets with iron dextran improves the piglets' hematological status, attenuates the induction of hepcidin expression, and minimizes the toxicity of the administered iron. (Am J Pathol 2010, 177:1233-1243; DOI: 10.2353/ajpath.2010.091020)

Iron deficiency is the most frequent cause of anemia in humans, ${ }^{1}$ and iron deficiency anemia (IDA) is most prevalent in the neonatal period and early childhood. ${ }^{2}$ Early postnatal iron deficiency is also a widespread phenomenon in other mammalian species. Indeed, IDA has Iong been recognized as a serious iron disorder in livestock, especially in suckling piglets. ${ }^{3}$ In contrast to humans and mice, the complex molecular regulation of body iron homeostasis ${ }^{4}$ has been the subject of few studies in pigs. ${ }^{5-7}$ Our knowledge of iron homeostasis in newborn pigs is mostly based on physiological concepts stemming from the early 1990s. Although the pig is a major biomedical mammalian model for human studies, ${ }^{8}$ its contribution to understanding the molecular pathophysiological mechanisms of human iron disorders has so far been small. ${ }^{9}$

The common reason for iron deficiency in newborn piglets is their rapid growth, particularly the increase in blood volume and the number of red blood cells (RBCs). Indeed, erythroid precursors in the bone marrow use most of the iron found in the plasma for the synthesis of hemoglobin. As sow's milk largely provides piglets with iron below their daily requirements, ${ }^{10}$ an exogenous source of iron is essential to prevent the reduction in RBC hemoglobin level. Intramuscular administration of large

Supported by Polish State Committee for Scientific Research (KBN) grant PBZ-KBN-093/P06/2003.

Accepted for publication May 20, 2010.

None of the authors disclosed any relevant financial relationships.

Supplemental material for this article can be found on http://ajp. amjpathol.org.

Address reprint requests to Dr. Paweł Lipiński, Department of Molecular Biology, Institute of Genetics and Animal Breeding, Polish Academy of Sciences, Jastrzebiec, ul. Postępu 1, 05-552 Wólka Kosowska, Poland. E-mail: P.Lipinski@ighz.pl. 
amounts of iron dextran (FeDex) on days 3 to 6 postpartum is current practice in the swine industry, ${ }^{3,11}$ and has been proven to rectify the hematological status of piglets. However, it seems unlikely that 100 to $200 \mathrm{mg}$ of iron (a commonly applied dose) given in a single injection to a piglet with only about 40 to $50 \mathrm{mg}$ of iron in its body at birth, ${ }^{12}$ is efficiently metabolized and detoxified. Moreover, high parenteral intake of supplemental iron may easily perturb the tight control of systemic iron metabolic processes. In this context, iron supplementation in piglets raises the question of the role of hepcidin (Hepc)-the systemic iron-regulatory hormone ${ }^{13}$ in the regulation of natural intestinal iron absorption, especially under conditions of morphological and functional rebuilding of the pig small intestinal mucosa that occurs within 21 days of birth. ${ }^{14}$

In this study we examined the expression of proteins involved in iron absorption and its regulation in newborn piglets during the first 2 weeks of life. We also tested a modified strategy for iron supplementation of anemic piglets and demonstrated its ability to improve their hematological status, regulate Hepc expression and minimize the toxicity of supplemental iron.

\section{Materials and Methods}

\section{Animals, Experimental Design, and Biological Sample Collection}

Experiments were conducted at the State pig farm (Dobrzyniewo, Poland). A total of 64 Polish Landrace $\times$ Pietrain piglets housed in standard conditions (approx. $70 \%$ humidity and a temperature of $22 \pm 2^{\circ} \mathrm{C}$ in standard cages with straw bedding) were used in the experiments. Piglets were taken from 11 litters delivered by 8 primipara and 3 multipara sows. During the 14-day experiment, sows were allowed to nurse their piglets. The number of piglets taken from each litter was from 4 to 8 depending on the litter size (10 to 16 piglets). They were allotted to one of three different experimental groups on the basis of body weight (b.w.) at the given experimental age (Figure 1): top) control piglets receiving no iron supplementation; middle) piglets intramuscularly injected in the neck with $100 \mathrm{mg} \mathrm{Fe} / \mathrm{kg}$ b.w. on day 3 postpartum (traditional sup-

\begin{tabular}{lllllll}
\hline & 1 & 1 & 1 & 1 & 1 \\
\hline & 4 & & 7 & & no Fe supplementation
\end{tabular}

$\downarrow 100 \mathrm{mg} \mathrm{Fe} / \mathrm{kg}$ b.w.

$+\begin{array}{lllll}1 & 1 & 1 & 1 & 1\end{array}$

\begin{tabular}{|c|c|c|c|}
\hline 3 & 4 & 7 & 10 \\
\hline
\end{tabular}

Figure 1. Experimental design scheme. Numbers indicate the age of piglets at which animals were sacrificed and biological samples collected. Boxed numbers indicate the days on which iron dextran supplementation was given. plementation); and bottom) piglets injected with $40 \mathrm{mg}$ $\mathrm{Fe} / \mathrm{kg} \mathrm{b.w.} \mathrm{on} \mathrm{day} 3$ and again on day 10 postpartum (modified supplementation). Iron was administered to piglets in the form of iron dextran (FeDex), a complex of ferric ions with low molecular weight dextran (Ferran 100, $10 \%$ solution, Vet-Agro, Lublin, Poland). Biological samples were collected from control piglets (group A) at the age of $1,2,4,7$, and 14 days and from iron-supplemented animals (groups B and C) at the age of 4,7 , and 14 days. Piglets were sacrificed for tissue sampling ( 5 to 6 animals for each time point) by $\mathrm{CO}_{2}$ inhalation and exsanguination. Immediately after death, liver fragments were excised and scrapings from the proximal duodenum were collected. Samples were frozen in liquid nitrogen and held at $-80^{\circ} \mathrm{C}$ until they were analyzed. The experiments described here were conducted in compliance with the European Union regulations concerning the protection of experimental animals. All procedures were approved by the third Local Ethical Commission in Warsaw (permission no. 46/2003).

\section{Hematological Analysis and Serum Iron}

Blood was drawn from piglets into heparinized tubing by direct cardiac puncture immediately after death. Erythrocyte counts and erythrocyte parameters as well as serum iron levels were determined using an automated SYMEX F820 Analyzer.

\section{Quantitative Hepatic Non-Heme Iron Measurement and Hepatic Iron Staining}

Quantification of the non-heme iron content of liver fragments $(100 \mathrm{mg}$ ) was performed using a BIOCHEMTEST (Polish Chemical Reagents, Gliwice, Poland) as described previously. ${ }^{15}$

Pig livers were fixed in Bouin's solution for 72 hours at $20^{\circ} \mathrm{C}$ and then stored in $70 \%$ ethanol before further preparation. After dehydration liver fragments were embedded in paraffin and cut into $5 \mu \mathrm{m}$ sections with rotation microtome (Microm HM 355S, MICROM GmbH, Germany). After mounting on glass slides, sections were deparaffinized, stained with Perls' Prussian blue, counterstained with nuclear red and analyzed under light microscope (Zeiss).

\section{Detection of Iron-Saturated Ferritin by Polyacrylamide Gel Electrophoresis}

The detection of ferritin was performed using a procedure for the identification of iron-binding proteins. ${ }^{16}$ Briefly, 80 $\mu \mathrm{g}$ samples of liver membrane extract were resolved by electrophoresis on 7\% polyacrylamide gels under nondenaturing conditions. The gels were then immediately placed in potassium ferricyanide solution (2\% in 50 $\mathrm{mmol} / \mathrm{L} \mathrm{Tris-HCl}, 100 \mathrm{mmol} / \mathrm{L} \mathrm{NaCl}, \mathrm{pH}$ 7.5), held in the dark for 15 minutes and then transferred to trichloroacetic acid/methanol solution. Iron-containing ferritin was visualized as blue bands in the native gels. To detect total 
Table 1. Oligonucleotide Primers Used for Real-Time Quantitative RT-PCR Analysis

\begin{tabular}{|c|c|c|c|c|}
\hline mRNA & Forward primer & Reverse primer & $\begin{array}{l}\text { Amplified } \\
\text { fragment } \\
\text { (bp) }\end{array}$ & Accession number \\
\hline Apex1 & 5'-GAATGCTGGCTTCACTCCACA-3' & 5'-AAAGGTGTAGGCATACGCCGT-3' & 115 & NM_001139471 \\
\hline Hamp & $5^{\prime}$-CGCAGCAGAAGATGCAGAT - $3^{\prime}$ & $5^{\prime}$-CGCAGCAGAAGATGCAGAT- $3^{\prime}$ & 115 & AF516143 \\
\hline Gapdh & $5^{\prime}$-GACCACAGTCCATGCCATCAC-3' & 5'-TCCACCACCCTGTTGCTGTAG-3' & 452 & NM_017370 \\
\hline $18 S$ & 5'-ATCCTTCGATGTCGGCTCTT-3' & $5^{\prime}-$ ACTAACCTGTCTCACGACGGTC-3' & 115 & CX059405.1 \\
\hline
\end{tabular}

ferritin level in the liver, hepatic membrane extracts (45 $\mu \mathrm{g} /$ lane) were separated on non-denaturing polyacrylamide gel electrophoresis (PAGE), and stained with Coomassie blue. To assist identification of Ft bands horse spleen holo- and apo-Ft were used as positive controls (electrophoresed on the same gel).

\section{Real-Time Quantitative Reverse Transcription-Polymerase Chain Reaction Analysis of mRNA Abundance}

Total cellular RNA was extracted from liver samples (20 mg) using TRIzol reagent (Invitrogen) according to the manufacturer's protocol. Two micrograms of total DNasetreated RNA were used for reverse transcription with MMLV reverse transcriptase (Promega) at $45^{\circ} \mathrm{C}$ for 1 hour. Real-time quantitative reverse transcription (RT)PCR of cDNAs derived from specific transcripts was performed in a Light Cycler (Roche Diagnostics, Mannheim, Germany) using the respective pairs of oligonucleotide primers indicated in Table 1. Amplification products were detected using SYBR Green I (Roche Diagnostics) as described previously. ${ }^{17}$ To confirm amplification specificity, the PCR products from each primer pair were subjected to melt curve analysis and agarose gel electrophoresis. Light Cycler 3.5 Software was used for data analysis. Expression was normalized relative to that of control transcripts encoding glyceraldehyde-3-phosphate dehydrogenase or 18S rRNA.

\section{Western Blot Analysis}

For the detection of duodenal divalent metal transporter 1 (DMT1) and ferroportin (Fpn), $100 \mu \mathrm{g}$ of membrane extracts from duodenal mucosa scrapings were done as previously described ${ }^{18}$ and were resolved by electrophoresis on $10 \%$ SDS-polyacrylamide gels. For the detection of L-ferritin, hepatic membrane $(45 \mu \mathrm{g})$ and cytosolic $(100 \mu \mathrm{g})$ extracts were prepared from the same liver samples ${ }^{18}$ and were resolved on 14\% SDS-polyacrylamide gels. Proteins were transferred from the gels to Hybond-ECL nitrocellulose membranes (Amersham Life Sciences, Little Chalfont, UK). The membranes were initially blocked by gentle agitation in Tris-buffered saline with $0.15 \%$ Tween 20 (TBST), containing 5\% fat-free dried milk, at room temperature for 1 hour. This was followed by overnight incubation at $4^{\circ} \mathrm{C}$ with the following primary rabbit antibodies: polyclonal antibodies raised against mouse Fpn and DMT1 (which cross-react with pig proteins ${ }^{7}$ and antisera raised against recombinant mouse light chain ferritin (L-Ft, a gift from Dr. Paolo Santambrogio, Milano, Italy) diluted in TBST containing 1\% dried milk. After briefly washing in TBST, the membranes were then incubated for 1 hour at room temperature with peroxidase-conjugated anti-rabbit secondary antibody (Santa Cruz Biotechnology, Santa Cruz, CA). Following a final wash in TBST, immunoreactive bands were detected using the ECL Plus enhanced chemiluminescence detection system (Amersham Life Sciences).

\section{Immunochemistry and Immunofluorescence}

Duodenal tissues were processed as previously described. ${ }^{18}$ Briefly, tissues were fixed in paraformaldehyde solution, dehydrated and followed by embedding in paraffin. Five-micrometer sections were deparaffinized in xylene and rehydrated for staining. For immunohistochemistry only, endogenous peroxidase activity in the deparaffinized sections was blocked with ethanol 70\%peroxide $1 \%$ solution. Sections were then incubated in PBS/100 mmol/L glycine, rinsed in PBS, and blocked for at least 1 hour at room temperature in PBS containing 1\% bovine serum albumin and 10\% normal goat serum (GIBCO). Incubation with the primary antibody diluted in blocking solution was performed in a wet chamber overnight at $4^{\circ} \mathrm{C}$. Immunohistochemical staining of fixed paraffin-embedded sections was performed using the peroxidase/antiperoxidase diaminobenzidine procedure (Dako). Sections were then counterstained with H\&E, and mounted in Permount. For immunofluorescence, after incubation with the primary antibodies, sections were further incubated with an anti-rabbit secondary antibody conjugated to Alexa488. Sections were then washed with PBS and directly mounted with antifading mounting reagent (Prolong Antifade kit P-7481 MolecularProbes) and processed for microscopy. Sections were visualized using epifluorescence microscope LEICA DM-IRM with a $\times 60$ oil immersion objective.

\section{DNA Isolation and Determination of 8-Oxo-7,8- Dihydro-2'-Deoxyguanosine Content}

DNA from pig livers was isolated and the content of 8-oxo-7,8-dihydro-2'-deoxyguanosine (8-oxodG) was determined using the high pressure liquid chromatography electrochemical detection technique as described previously. ${ }^{19}$ 

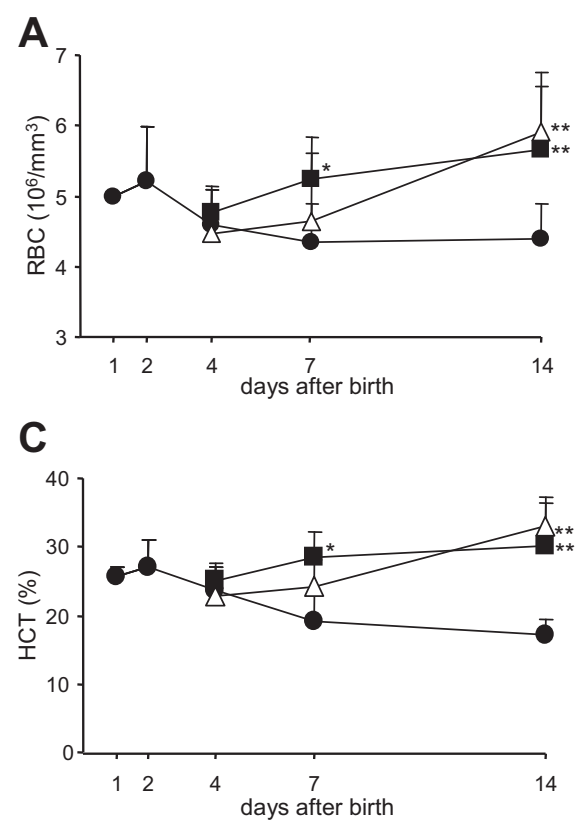

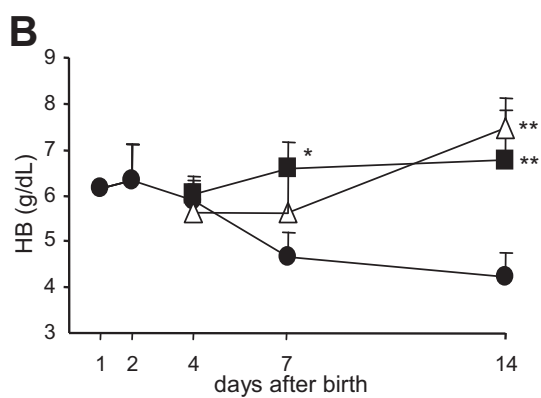

D

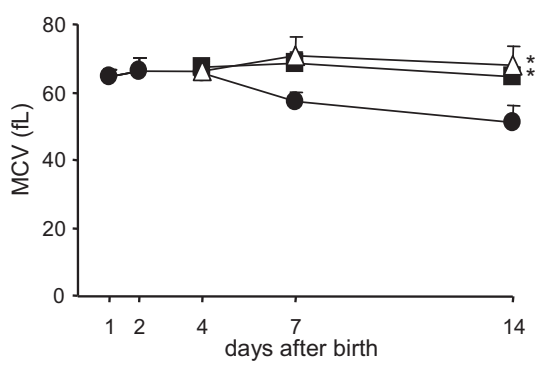

Figure 2. Hematological parameters of control and FeDex-supplemented piglets. Blood cell indices were determined for 5 to 6 piglets from each group at each time point. Red blood cell (RBC) count (A); Hemoglobin (Hb) concentration (B); Hematocrit (HCT) value (C) and mean cell volume (MCV) (D). Values are expressed as the mean $\pm \mathrm{SD}$. Asterisks indicate significant differences $\left({ }^{*} P<0.05,{ }^{* * *} P<0.01\right)$ in comparison with control values at the given day after birth.

\section{DNA Repair Activity Assay}

The DNA repair activities of APEX and OGG1 in piglet livers were measured by the nicking assay. ${ }^{20}$ Liver fragments were homogenized with four volumes of the appropriate buffer, disrupting the cells by sonication. Cell debris was removed by centrifugation, and the supernatants used as the source of DNA repair enzymes. Oligonucleotides (40-mers) containing a single 8-oxo-guanine or an apurinic/apyrimidinic (AP) site at position 20 in the sequence 5'-d(GCTACCTACCTAGCGACCTXCGACTGTCCCACTGCTCGAA)-3'(Eurogentec Herstal, Belgium or MetaBion, Germany)—where X indicates either 8-oxoguanine or an AP-site-were ${ }^{32} \mathrm{P}$-labeled at the $5^{\prime}$-end and purified on Micro-Bio-Spin-30 chromatography columns as described by the manufacturer (Bio-Rad). These were annealed, at a twofold molar excess, to complementary unlabeled oligodeoxynucleotides, and the formation of duplexes was verified by native $20 \%$ polyacrylamide gel electrophoresis. The oligodeoxynucleotide duplexes were incubated with the tissue extracts for 1 hour at $37^{\circ} \mathrm{C}$, and the reaction products separated by denaturing $20 \%$ polyacrylamide gel electrophoresis. A digital image of the separated radioactive products was prepared by scanning the gel using a phosphorimager and the band intensities quantified with ImageQuant software (Molecular Dynamics, version 5.2). From each data set, a Michaelis-Menten curve was plotted and the enzyme activity calculated. All activity assays were performed at least in triplicate. Two control reactions were used in each experiment: 1) a negative control in which the nontreated labeled oligonucleotide duplex was subjected to denaturing PAGE to show if any nonspecific degradation had occurred during the procedure; 2) a positive control in which the oligonucleotide duplex was digested with an excess of the appropriate purified DNA glycosylase.

\section{Statistical Evaluation}

Data are presented as mean values $\pm \mathrm{SD}$. Assumptions of normality and equal variance were tested before parametric analyses based on two-way analysis of variance (factors: time and dose), with individual comparisons made using the Student-Neuman-Keuls post hoc test. When these assumptions could not be confirmed, nonparametric analysis was performed using Kruskal-Wallis analyses of variance, followed by the Mann-Whitney Utest. Statistical significance was considered at $P<0.05$ and $P<0.01$. Statistica for Windows software (version 5.0) was used for the statistical analysis.

\section{Results}

\section{Iron Supplementation Prevents a Decrease in the RBC Indices of Newborn Piglets}

Evaluation of the hematological parameters of 1- and 2-day-old piglets clearly showed that these animals were verging on borderline anemia (Figure 2, A-D). Without iron supplementation their hematological status gradually and significantly worsened. On day 14 after birth, low values for RBC count, hematocrit percentage, hemoglobin concentration and mean erythrocyte volume together with decreased serum iron level (Figure 3A) indicated the occurrence of severe iron deficiency anemia. Both iron treatments efficiently prevented the deterioration of the hematological status of newborn piglets and contributed to the recovery of animals from the pre-anemic state observed by postnatal day 2. The first dose of iron $(40 \mathrm{mg}$ $\mathrm{Fe} / \mathrm{kg}$ b.w.) given to piglets according to the split supplementation protocol (Figure 1) was at least as effective as the single high dose (100 mg Fe/kg b.w.), used in traditional supplementation, at improving the values of the 
A

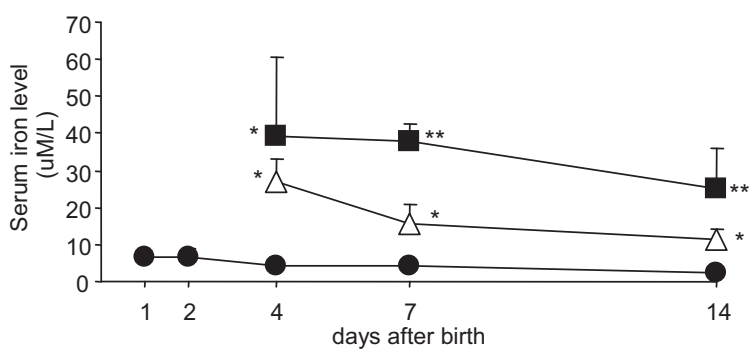

B

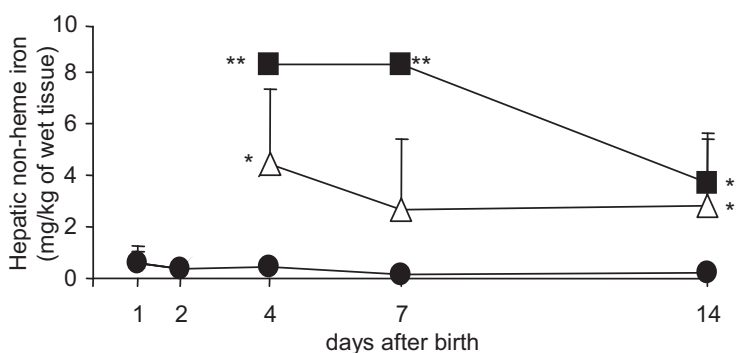

C

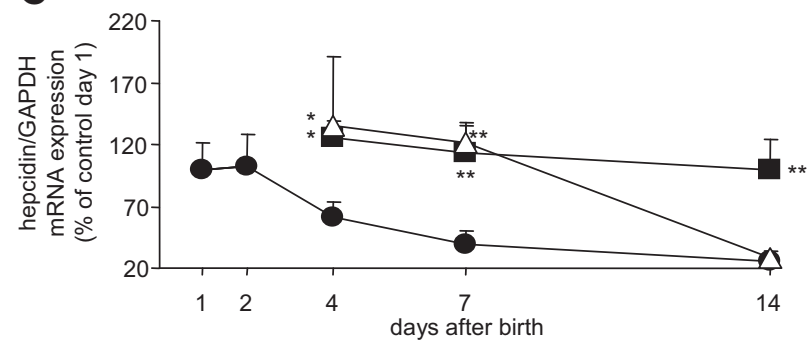

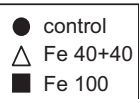

Figure 3. Correlation between serum iron level, hepatic non-heme iron content and hepatic hepcidin mRNA abundance in control and FeDexsupplemented piglets. Serum iron levels (A) and hepatic non-heme iron content (B) in control and FeDex-supplemented piglets. Values are expressed as the mean \pm SD for serum and liver samples obtained from 5 to 6 piglets at each time point. C: Hepc mRNA abundance in piglet livers measured by real-time RT-PCR analysis. Each point represents the mean \pm SD of two amplification reactions, performed on a single cDNA sample reversetranscribed from separate RNA isolated from 5 to 6 different piglets. Values were normalized against the level of transcript encoding glyceraldehyde-3phosphate dehydrogenase. The level of Hepc mRNA level in control piglets (day 1) was assigned the value of $100 \%$. Asterisks indicate significant differences $\left({ }^{*} P<0.05,{ }^{* *} P<0.01\right)$ in comparison with control values at the given day after birth.

hematological parameters up to day 7. The second injection of iron, given according to the modified protocol, sustained a gradual increase in the values of all analyzed RBC parameters. On day 14 after birth, piglets from both groups that had received supplemental iron showed no significant difference in their hematological profiles.

\section{Relationship Between Iron Status of Piglets and Hepatic Hepc mRNA Level}

Iron was the first biological factor shown to induce hepcidin expression ${ }^{21}$ and there is experimental evidence that both high iron saturation of plasma transferrin ${ }^{22}$ and hepatic iron loading ${ }^{21}$ stimulate Hepc synthesis. The relationship between hepatic non-heme iron content, serum iron level and hepatic Hepc mRNA abundance was examined in control and iron-supplemented piglets. Despite their low hepatic iron content and serum iron level, 1- and 2-day-old piglets showed relatively high hepatic Hepc transcript abundance (Figure 3, B and C), similar to that of 200-day-old adult pigs with a much higher liver iron content (see Supplemental Figure 1S at http://ajp. amjpathol.org). The observed decrease in hepatic iron content and serum iron level in control piglets after day 2, was associated with a parallel decline in Hepc mRNA levels (Figure 3C). Iron supplementation on day 3 (either 40 or $100 \mathrm{mg} \mathrm{Fe} / \mathrm{kg} \mathrm{b.w.)} \mathrm{efficiently} \mathrm{improved} \mathrm{the} \mathrm{iron}$ status of piglets and slightly elevated liver Hepc expression 24 hours after administration (ie, on day 4). On postnatal day 7 the piglets injected with $100 \mathrm{mg} \mathrm{Fe} / \mathrm{kg}$ b.w. had a greater hepatic iron content and higher serum iron level than those supplemented with $40 \mathrm{mg} \mathrm{Fe} / \mathrm{kg}$ b.w., but there were no noticeable differences in Hepc mRNA level between these two groups. During the final stage of the experiment, pigs supplemented with iron in the traditional manner still maintained a high level of Hepc expression, whereas animals supplemented by the split protocol showed only residual expression similar to that of anemic piglets on day 14 (Figure 3C).

\section{Levels of DMT1 and Fpn in the Duodenal Mucosa of Newborn Piglets}

DMT1 and Fpn protein levels in membrane extracts from duodenal mucosa scrapings were measured in control anemic piglets to determine their molecular potential for iron absorption. On day 1 after birth both DMT1 and Fpn were barely detectable, and on day 2 these two iron transporters were expressed at a very low level (Figure 4). The expression of DMT1 increased dramatically by day 4 and thereafter remained at a slightly lower level. A similar although less pronounced compared with DMT1 increase in expression was observed in the case of Fpn. One can note some differences between day 4, 7, and 14 in apparent molecular weigh of both DMT1 and Fpn. Such changes in protein migration in gel electrophoresis may reflect some changes in post-translational modifications of the protein species detected. Indeed, duodenal (adult) pig Fpn was shown to be post-translationally modified by complex $\mathrm{N}$-linked glycosylation. ${ }^{7}$ At day 7 , when both Fpn and DMT1 levels are high, we looked at the subcellular localization of the two transporters in the duodenum of control piglet. Using both immunofluorescence technique or classical immunohistochemistry on duodenal section, we observed the opposite and complementary localization of DMT1 and Fpn (Figure 4B). DMT1 was strongly detected in the villi at the apical site of enterocytes corresponding to the brush border whereas Fpn was found exclusively at the basolateral membrane of absorptive enterocytes. This localization in the piglet duodenum is in accordance with previous localization observed in mouse duodenum and attested of the specificity of the antibodies used. A comparison of 


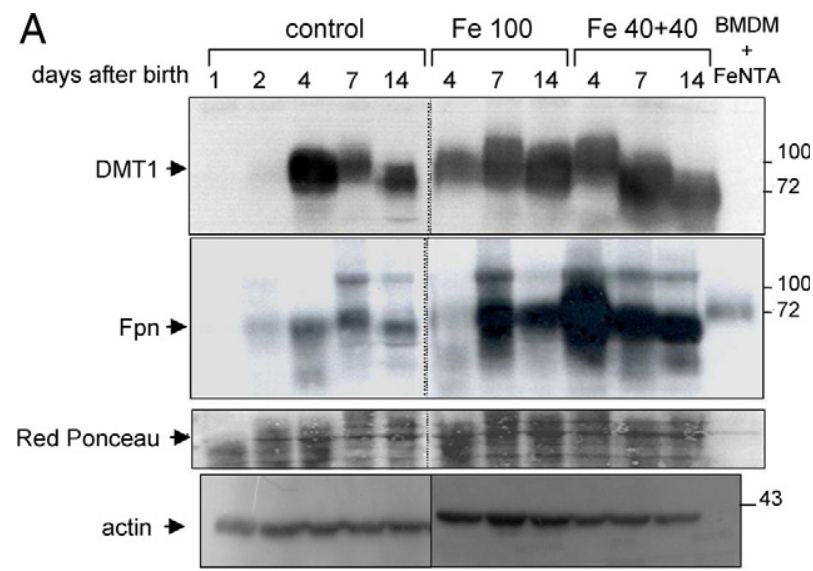

B
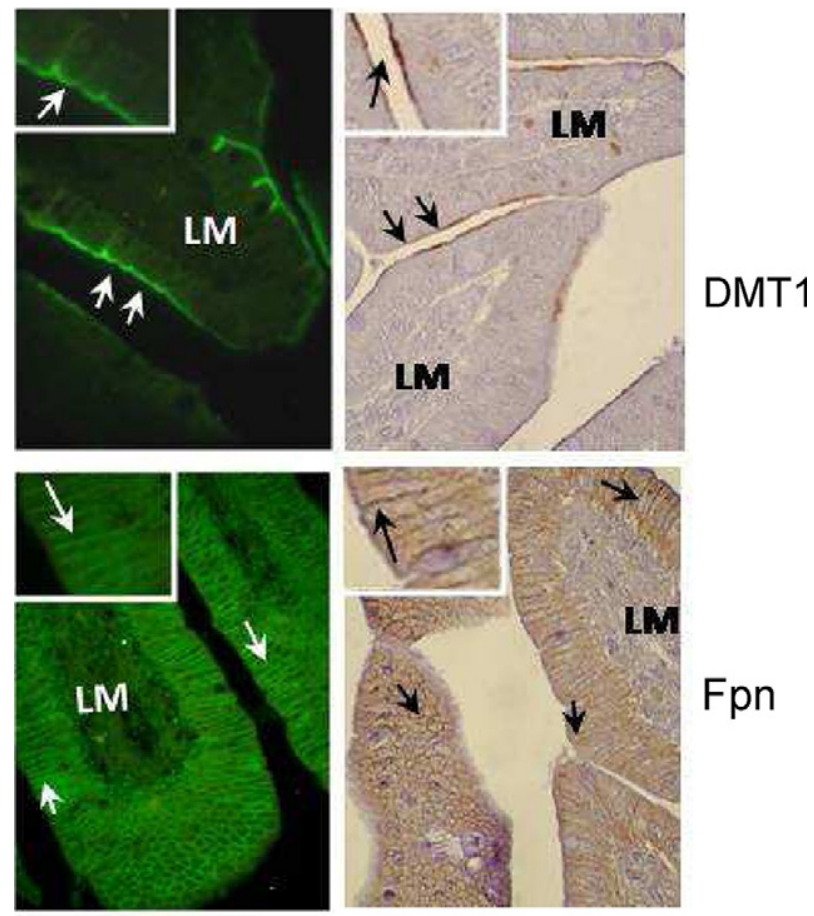

control, day 7

Figure 4. Duodenal ferroportin (Fpn) and divalent metal transporter (DMT1) protein levels in anemic non-supplemented and FeDex-supplemented piglets. A: DMT1 and Fpn levels in duodenum membrane protein extracts (100 $\mu \mathrm{g} /$ lane) as assessed by Western blotting. Ponceau Red staining of transferred proteins is shown to confirm similar loading. In addition, identical blot reacted with actin antibody is shown to confirm equivalent loading. The presented data are representative of Western blotting analyses of duodenum membrane extracts obtained from three piglets at each time point. B: Cellular localization of DMT1 and Fpn in duodenal tissues sections of control piglet at day 7. By immunofluorescence (left panels, green) of immunohistochemistry (right panels, brown staining) DMT1 and Fpn were strongly detected at the apical brush border and basolateral membrane respectively (see arrows and high magnification inset). LM, luminal membrane.

the duodenal Fpn and DMT1 protein levels in piglets supplemented with FeDex and in controls was next performed using western analysis (Figure 4A). Such analysis revealed an unexpected up-regulation of Fpn in animals receiving intramuscular iron injections (Figure 4A, rightmiddle panel). Moreover, Fpn levels tended to be higher in piglets supplemented twice with $40 \mathrm{mg}$ Fe/kg b.w. than in traditionally-supplemented animals, especially on day 4 after birth. In contrast to the control piglets, duodenal Fpn expression in FeDex-supplemented animals (Figure 4A, right-middle panel) showed no relationship with hepatic hepcidin mRNA levels (Figure $3 C)$. Considering that the Fpn mRNA bears an ironresponsive element within its $5^{\prime}$ UTR, ie, a binding site for iron regulatory proteins, ${ }^{23}$ the possibility that Fpn is up-regulated in FeDex-supplemented piglets by an iron-mediated posttranscriptional mechanism was examined. However, a comparison of the iron-responsive element-binding activity of iron regulatory protein-1 in the duodenum showed no difference between piglets from the three experimental groups (see Supplemental Figure 2S at http://ajp.amjpathol.org)

\section{Histological Evidence of Massive Hepatic Iron Accumulation in Piglets Supplemented with a High Amount of Iron Dextran}

Microscopic analysis of liver sections stained for nonheme iron with Perls' Prussian blue showed heavy iron deposits in the Kupffer cells of piglets supplemented with $100 \mathrm{mg} \mathrm{Fe} / \mathrm{kg}$ b.w. (Figure 5A, bottom panel). This macrophagic iron accumulation was observed 24 hours after the injection of FeDex (Day 4) and was maintained until the end of the experiment (Day 14). In comparison, the iron accumulation in the livers of piglets supplemented twice with $40 \mathrm{mg} \mathrm{Fe} / \mathrm{kg}$ b.w. was definitely smaller following the first FeDex injection and had decreased slightly when examined again on postnatal days 7 and 14 despite the second FeDex injection on day 10 (Figure 5A, middle panels). In anemic piglets receiving no supplemental iron, hepatic non-heme iron was not detected by Prussian blue staining (Figure 5A, top panels). To identify the main non-heme iron-containing compounds in the livers of piglets supplemented with FeDex, liver membrane extracts were resolved on polyacrylamide gels and specific staining was used to detect non-heme iron. It was found that FeDex constituted a major part of the hepatic iron in the traditionally-supplemented piglets (Figure 5B, upper panel). The amount of FeDex successively decreased throughout the experimental period but was still present in the livers of 14-day-old piglets. In the liver extracts from piglets given the modified supplementation protocol only a low concentration of FeDex was detected at day 4 after birth, following the first injection of $40 \mathrm{mg} \mathrm{Fe} / \mathrm{kg}$ b.w. At later time-points, FeDex was undetectable in the liver of piglets from this group. The major endogenous iron-containing protein found in the liver is ferritin. Iron ferritin saturation in hepatic membrane extracts was detected by specific staining of ferritin-bound iron after separation by PAGE. ${ }^{16}$ It was found to be very high (comparable with that of purified horse spleen holoferritin, Figure 5B, upper panel) in piglets injected with the $100 \mathrm{mg} \mathrm{Fe} / \mathrm{kg} \mathrm{b.w}$. dose. However, iron bound to ferritin was not detected in the extracts from piglets receiving two injections of $40 \mathrm{mg} \mathrm{Fe} / \mathrm{kg} \mathrm{b.w}$. or in those of animals receiving no supplemental iron. Semiquantitative analysis of the total content of ferritin in hepatic membrane extracts performed by Coomassie blue staining 
A day 4
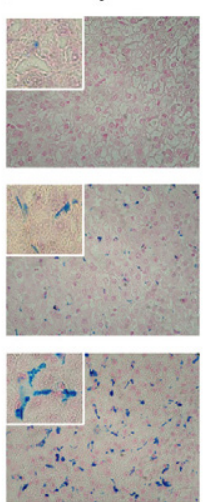

B
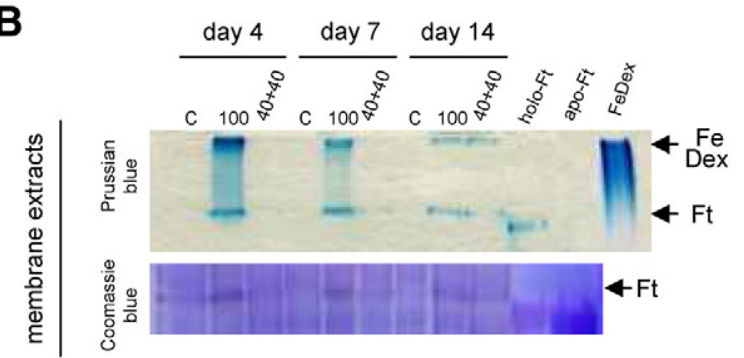

C
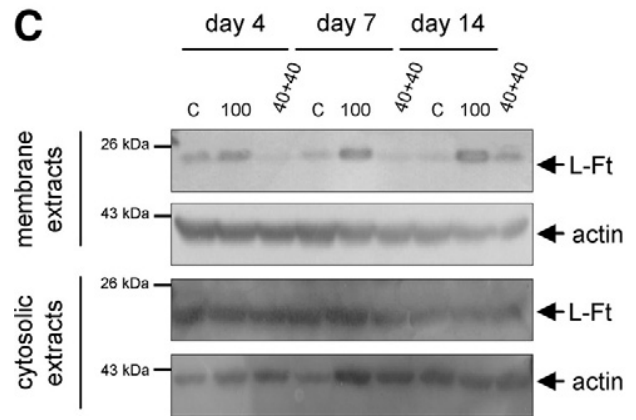

Figure 5. Hepatic iron status/distribution and ferritin level in control and FeDex-supplemented piglets. A: Histological examination of iron loading in liver samples. Non-heme iron deposits were detected by staining with Perls Prussian blue and the cells counterstained with nuclear red. Blue staining was present exclusively in the Kupffer cells of FeDex-supplemented piglets. A high magnification of Kupffer cells presenting deposits of iron or their absence in FeDex-supplemented piglets and control animals, respectively is shown (insets in the top, middle, and bottom right panels). B (upper panel): Potassium ferricyanide staining of iron-bound ferritin and iron dextran in hepatic membrane extracts from FeDex-supplemented piglets. Iron saturated horse spleen holo-ferritin (holo-Ft) and iron dextran (FeDex) were used as positive controls. Horse spleen apo-ferritin (apo-Ft) was used as negative control. B (bottom panel): Membrane proteins from liver (45 $\mu \mathrm{g} /$ lane) were separated on non-denaturing PAGE (as in B), and stained with Coomassie blue. To assist identification of Ft bands horse spleen holo- and apo-Ft were used as positive controls (electrophoresed on the same gel). C: L-Ft levels were assessed in membrane (upper panel) and cytosolic (lower panel) extracts by Western blotting as described in the Materials and Methods section. To assist identification of L-Ft bands, recombinant mouse L-Ft (rL-Ft, a gift from Dr Paolo Santambrogio) was electrophoresed on the same gel. Blots were also reprobed with monoclonal anti- $\beta$-actin antibody as a loading control The analyses were performed using liver sections and extracts obtained from three to four different piglets, and representative results are presented.

after separation by PAGE shows high levels of $\mathrm{Ft}$ in piglets receiving a single high dose of FeDex (Figure 5B, lower panel). Similarly, more detailed and precise Western blot analysis of L-Ft (a dominant form of ferritin in the liver) in hepatic membrane extracts reveals increased amount of light chain Ft in this group of piglets (Figure 5C,

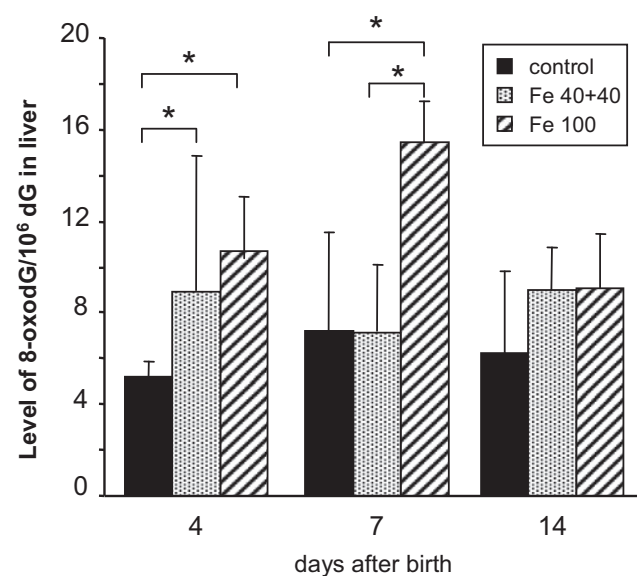

Figure 6. Levels of 8-oxodG in liver DNA of control and FeDex-supplemented piglets. Values are expressed as the mean \pm SD for analyses of liver DNA samples obtained from five to six piglets at each time point. Significant differences of ${ }^{*} P<0.05$.

upper panel). Surprisingly, Western blot analysis showed no major changes in the amount of cytosolic L-Ft between piglets from different groups (Figure 5C, lower panel).

\section{Hepatic Level of 8-oxodG Is Increased in FeDex Supplemented Piglets}

Numerous studies have highlighted the role of iron in oxidative stress and damage to DNA (24 and the references therein). The base modification 8-oxodG is regarded as a good biomarker of oxidatively damaged DNA (for review see 25). The hepatic level of this oxidative DNA adduct was analyzed in piglets receiving supplemental iron and control animals. There was a statistically significant elevation of the level of 8-oxodG in DNA isolated from the livers of 4-day-old iron-supplemented piglets (ie, 1 day after injection of either 100 or 40 mg $\mathrm{Fe} / \mathrm{kg} \mathrm{b.w.)} \mathrm{in} \mathrm{comparison} \mathrm{with} \mathrm{nonsupplemented} \mathrm{ani-}$ mals (Figure 6). Supplementation with a single high dose of iron resulted in a higher although nonsignificant increase in the 8-oxodG level when compared with the piglets on the split supplementation protocol. On day 7 this difference was highly statistically significant as the hepatic level of 8-oxodG in piglets supplemented with $100 \mathrm{mg} \mathrm{Fe} / \mathrm{kg}$ b.w. was about double the value found in both control piglets and those supplemented with the lower dose of $40 \mathrm{mg} \mathrm{Fe} / \mathrm{kg} \mathrm{b.w.} \mathrm{By} \mathrm{postnatal} \mathrm{day} 14$ the difference between the control and supplemented piglets was no longer significant although the latter animals still showed slightly raised hepatic levels of 8-oxodG.

\section{Modulation of the Expression and Activity of DNA Repair Enzymes in the Liver of Newborn Piglets}

Transcription of some DNA repair genes, including those participating in 8-oxodG repair such as APEX, MutT, and to a lesser extent OGG1, is greatly stimulated by reactive oxygen species (for review see 26). The administration of 

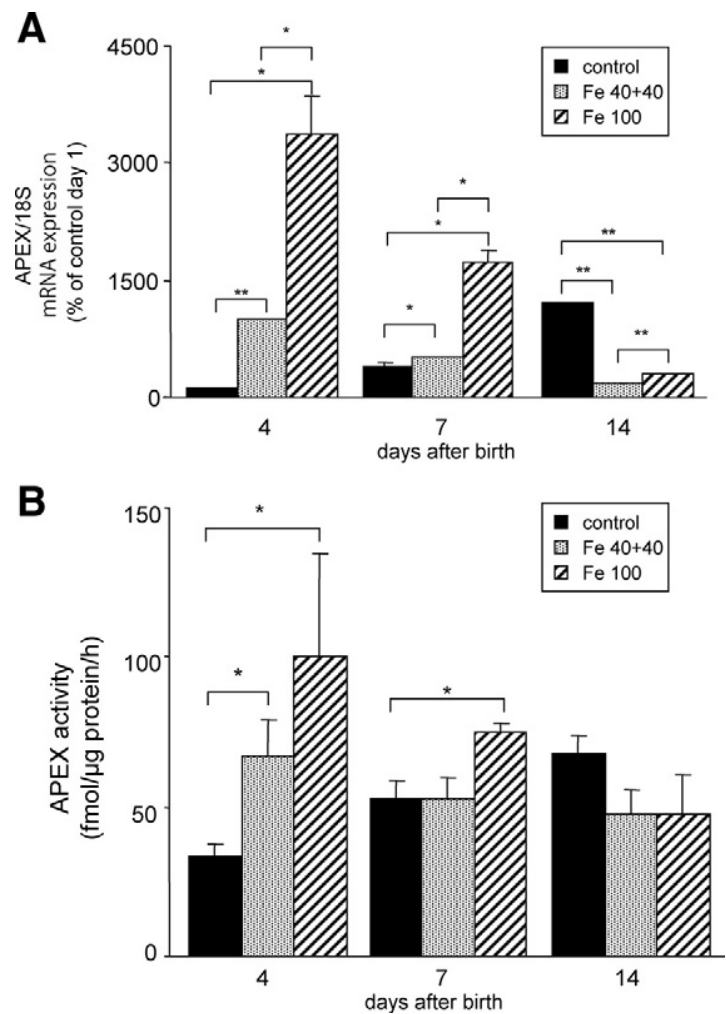

Figure 7. APEX mRNA level (A) and AP-site incision activity (B) in the livers of control and FeDex-supplemented piglets. A: APEX transcript abundance in piglet livers was measured by real-time RT-PCR analysis. Reactions and data presentation are as described in the legend to Figure $3 \mathrm{C}$ except that the values were normalized against the level of $18 \mathrm{~S}$ rRNA. The level of APEX mRNA in control piglets (day 1) was assigned the value of $100 \%$. B: APEX DNA repair activity in piglet livers was measured by the nicking assay. Values are expressed as the mean \pm SD for analyses of liver DNA samples obtained from five to six piglets at each time point. Significant differences of ${ }^{*} P<0.05,{ }^{* *} P<0.01$.

FeDex to 3-day-old piglets was found to increase the level of APEX transcript in the liver independently of the schedule of administration (Figure 7A). When examined the day after administration, the single high FeDex dose had caused an increase in APEX transcription of over 32-fold and this stimulation persisted for at least the next four days (until day 7). However, by day 14 the APEX mRNA level had fallen below that found in nonsupplemented animals. Administration of the low and split iron dose resulted in a moderate (9.5-fold) induction of APEX mRNA. This induction was only observed on the day after FeDex administration, and by postnatal day 7 . The level of APEX transcript had decreased to the control value, and it had dropped below the control by day 14 (Figure 7A).

Since the activity of DNA repair enzymes may be regulated via different modes, we verified the effect of iron administration on the ability of the piglet liver extracts to cleave abasic sites. The day after the administration of the single high iron dose, the rate of cleavage of the oligodeoxynucleotide with a single abasic site had increased by about threefold (Figure 7B). This APEX activity then decreased gradually: it was still higher than in control animals on day 7 , but by day 14 it had dropped to below the control value. The lower, split dose of iron

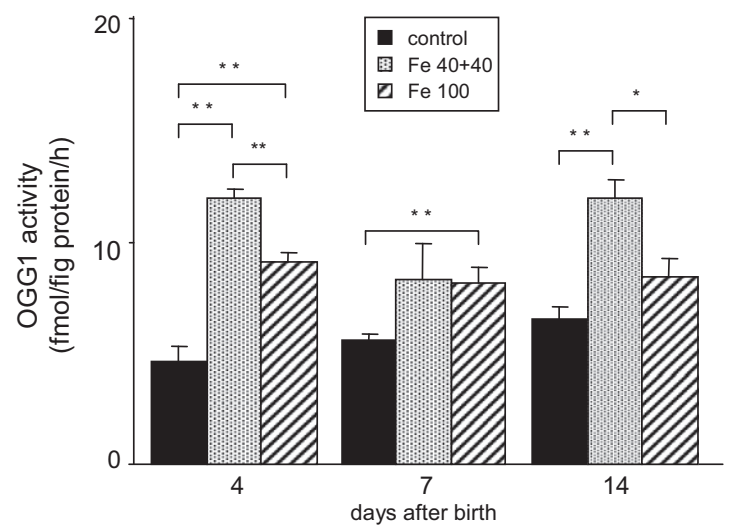

Figure 8. 8-OxoGua excision activity in the livers of control and FeDexsupplemented piglets. OGG1 DNA repair activity in piglet livers was measured by the nicking assay. Values are expressed as the mean \pm SD for analyses of liver DNA samples obtained from five to six piglets at each time point. Significant differences of ${ }^{*} P<0.05,{ }^{* *} P<0.01$.

resulted in moderate and short-term APEX induction. The APEX activity was increased on the day after administration of the first dose, but was similar to the control values when examined on day 7 , and on day 14 , following the second dose.

The rate of 8-oxoG excision was also increased on the day after the administration of both doses of iron (Figure 8). Unexpectedly, the excision rate was higher in animals receiving the lower, split dose of FeDex, than in those receiving the single high dose. This was observed on day 4 and day 14, while on postnatal day 7, 8-oxoG excision activities were the same in both groups receiving iron supplementation, but they were still higher than in the control animals.

\section{Discussion}

IDA is probably the most prevalent micronutrient deficiency disorder in pigs and the most frequent form of anemia in mammals. ${ }^{3}$ The goal of the present study was to investigate the molecular regulation of iron absorption in IDA of neonatal piglets and to evaluate the benefits and risks of iron supplementation during the first 2 weeks after birth. The major etiological factors in pig IDA include low fetal iron stores, the high intensity of piglet growth, increasing litter size, and inadequate levels of iron in sow's milk. 3,27 However, little is known about the molecular regulation of iron absorption in suckling piglets during the immediate postpartum period. A number of studies have demonstrated the limited efficacy of dietary iron supplements in preventing pig IDA. ${ }^{28,29}$ The poor responsiveness of piglets to oral iron therapy strongly suggests some immaturity in the molecular machinery of duodenal iron absorption. Indeed, we have demonstrated that the expression of DMT1 and Fpn, two iron transporters critical for intestinal iron absorption, was barely detectable during the two first days of life and only started to increase at around the fourth day after birth. This timing coincides with the replacement of "fetal-type" enterocytes with the "adult-type" lacking the apical canalicular system responsible for the absorption of bioactive pro- 
teins and peptides. ${ }^{30}$ After day 4 , the two transporters are strongly expressed at their known site of activity in enterocytes Similar DMT1 and Fpn expression profiles have also been reported during early infant development in rat $^{31}$ and mouse pups. ${ }^{32}$ Taken together these data suggest that mechanisms of iron absorption that function in adults may not be fully developed in neonatal mammals. Alternatively, in the case of Fpn its low expression in piglets observed in the 2 days after birth may be a consequence of high hepatic Hepc expression, which is consistent with the current concept of Fpn down-regulation by Hepc. ${ }^{33}$ High Hepc expression in newborn piglets is puzzling considering their low hepatic iron content. Interestingly, high levels of Hepc mRNA have previously been observed in rats ${ }^{21}$ and mice ${ }^{34}$ in the perinatal period. It is known that the birth process initiates an acute phase response in the healthy fetus-newborn infant with increased circulating levels of interleukin-6, ${ }^{35}$ a wellknown inflammatory cytokine that is responsible for the induction of Hepc during inflammation. ${ }^{36}$ Surprisingly, in contrast to anemic piglets, the Hepc-Fpn regulatory axis in FeDex-supplemented animals appeared to be nonfunctional. A possible explanation for this phenomenon may be that systemic regulation of $\mathrm{Fpn}$ is immature in newborn piglets., ${ }^{2,37}$ Alternatively, there is evidence showing that in contrast to hepatic Fpn, duodenal Fpn is poorly sensitive to the rise in Hepc levels. ${ }^{38,39}$ Finally, although Hepc mRNA expression is most preferred in animal studies, its correlation with circulating bioactive Hepc (a 25-amino acid peptide) levels is largely unknown. Several suitable serum Hepc assays have been developed mostly for humans. ${ }^{40,41,42}$ However, recent results of the first international round robin for the quantification of urinary and plasma hepcidin assays show that more work is needed for the use of Hepc plasma evaluation in experimental and clinical application. ${ }^{43}$

Several cases of iron toxicity have been reported on the commonly practiced administration of large amounts of FeDex in a single intramuscular injection. ${ }^{28,44}$ To optimize efficacy in correcting IDA, and minimize iron toxicity and Hepc synthesis we have tested a split supplementation protocol by which to give exogenous iron to piglets at an early age. Our results clearly show that this modified parenteral FeDex therapy satisfies all of the requirements of a method for administering iron to piglets. First, the modified iron supplementation efficiently prevents the decrease in the main RBC indices. Second, under this split protocol the stimulatory effect of parenteral iron on Hepc mRNA expression is completely lost after the second dose of FeDex, whereas the traditional use of a single high dose results in a sustained hepatic Hepc mRNA increase. In accordance with the proposed role of circulating holo-transferrin in signaling the increase in Hepc expression in hepatocytes, ${ }^{45}$ it is tempting to propose that the delayed drop in hepatic Hepc mRNA level in piglets receiving split supplementation is directly linked to the continuously decreasing serum iron concentration. In this group of piglets, iron released from FeDex ingested by Kupffer cells (known to accumulate iron after FeDex injection in animals) ${ }^{46}$ appears to be rapidly recy- cled into the circulation to be preferentially used in the process of enhanced erythropoiesis and is provided in limited amounts to the parenchyma of the liver to induce Hepc synthesis. Indeed, the analysis of non-heme iron content and its cellular distribution in the liver of piglets receiving two doses of $40 \mathrm{mg} \mathrm{Fe} / \mathrm{kg}$ b.w. showed no substantial iron accumulation in liver cells. In contrast, Kupffer cells from traditionally-supplemented piglets displayed strong Prussian blue iron staining throughout the experimental period. Moreover, the pattern of molecular iron distribution provided evidence of high ferritin saturation with iron and the persistence of large amounts of intact FeDex. These histological data corroborate the findings of postmortem examination of the visceral, limb and neck lymph nodes. The lymph nodes in piglets supplemented with $100 \mathrm{mg} \mathrm{Fe} / \mathrm{kg}$ b.w. were much more biger and darker than those of piglets supplemented with 40 $\mathrm{mg} \mathrm{Fe} / \mathrm{kg}$ b.w. (data not shown). Accordingly, after i.m. FeDex injection iron enters the RES through the lymphatic circulation. A detailed analysis of Ft expression at the protein level shows high amount of iron-rich Ft in hepatic membrane extracts (supposed to contain lysosomal fraction) in piglets receiving a single high dose of FeDex, and no major difference in the level of cytosolic L-Ft. We hypothesize that in piglets injected with a single high dose of FeDex, large amount of iron released from this compound is efficiently detoxified by cytosolic $\mathrm{Ft}$, which is then rapidly taken into the lysosomes and degraded. It has been shown previously that iron-laden $\mathrm{Ft}$ molecules are preferentially eliminated from the cytosol and are faster taken up by lysosomes than normally iron-saturated molecules. ${ }^{47}$ The appearance of siderosomes (heavily iron-laden terminal lyzosomes) has been also demonstrated under conditions of iron overload - in rats given injections of iron dextran. ${ }^{48}$ Vast amount of solubilized iron from the ferric core of degraded Ft molecules could then induce intralysosomal iron-catalyzed oxidative reactions with damage to lysosomal membranes and leakage of destructive contents.

When in excess, iron is toxic because it generates the hydroxyl radical that reacts readily with biological molecules. Therefore, besides efficacy in correcting iron deficiency, one of the main criteria for selecting an iron supplementation protocol is that the supplemental iron should produce minimal toxicity. The oxidatively damaged DNA exacerbated by iron may be involved in the development of various pathological conditions (for review see 25, 49). Considering the striking differences in the hepatic non-heme iron content found in piglets from the three experimental groups, 8-oxodG was used as a biomarker of iron-induced oxidative stress in piglet livers. In our previous study we observed a strong correlation between iron content and levels of the oxidatively modified nucleoside in lymphocytes' DNA. ${ }^{24}$ The results of the present study also showed that points with the highest iron concentrations were related to the highest 8-oxodG levels. Furthermore, the modified iron supplementation was linked with a significantly smaller increase in 8-oxodG level in hepatic DNA compared with the traditional protocol. These findings strongly suggest that the split supplemen- 
tation of piglets with FeDex minimizes the potential of parenteral iron therapy to induce oxidative stress and DNA damage.

The extent of oxidatively damaged DNA is also dependent on the efficiency of DNA repair systems. The main pathway to remove 8-oxoGua from DNA is base excision repair (BER) initiated predominantly by OGG1 glycosylase. ${ }^{50}$ The activity of this enzyme is regulated by the next enzyme of the BER pathway: abasic sites endonuclease (Apex1). We observed an iron-triggered increase in levels of Apex 1 mRNA, which was particularly large following supplementation with the single high dose of iron. The transcription of APEX is known to be induced by oxidative stress. ${ }^{51}$ The ability of liver extracts to incise abasic sites was also increased in the livers of animals supplemented with iron, and this induction was higher in piglets receiving a single high dose of FeDex than when two smaller doses were given in the split protocol. The increase in APEX activity was transient and decreased to the control level on the seventh day of life in animals receiving a split dose of iron, but remained high in the group receiving the single dose. Similarly, an increased rate of 8-oxoGua excision was observed up to day 14 of the experiment in the livers of piglets supplemented with FeDex by the two different protocols. OGG1 is a housekeeping gene, 52 which may also be induced by oxidative stress. ${ }^{53}$ On postnatal days 4 and 14, higher 8-oxoGua excision activity was observed in animals treated with the split dose than with a single high dose of FeDex. One possible explanation is inhibition of OGG1 activity by inflammation caused by iron overload in the livers of piglets treated with the single high dose of iron. OGG1 activity is inhibited by an inflammatory process that was shown to be produced in response to the administration of a high iron dose in rats. ${ }^{54}$ In this study, piglets supplemented with a single high dose of FeDex were found to be particularly prone to unbalanced DNA repair activity. Since the differences in the repair rate of the two studied enzymes were smaller following a split dose of iron, this modified supplementation protocol may be beneficial.

In summary, the results of this study provide evidence that the molecular regulation of iron absorption in newborn anemic piglets is immature, which possibly explains the poor responsiveness of these animals to oral iron therapy. Our findings also show that parenteral iron supplementation has to be based on a tight balance between possible benefits and risks: the efficacy of iron supplements in combating anemia and the generation of oxidative damage due to iron excess.

\section{Acknowledgments}

We thank John Gittins for critical reading the manuscript before submission. We are grateful to Dr. Murat Saparbaev (UMR 8126 C.N.R.S, University Paris-Sud, Institute Gustave Roussy, Villejuif, France) for a kind gift of DNA repair enzymes, OGG1 and APEX1.

\section{References}

1. Clark SF: Iron deficiency anemia: diagnosis and management. Curr Opin Gastroenterol 2009, 25:122-128

2. Collard KJ: Iron homeostasis in the neonate. Pediatrics 2009, 123:1208-1216

3. Svoboda M, Drabek J: Iron deficiency in suckling piglets: etiology, clinical aspects and diagnosis. Folia Vet 2005, 49:104-111

4. Andrews NC: Forging a field: the golden age of iron biology. Blood 2008, 112:219-230

5. Sang Y, Ramanathan B, Minton JE, Ross CR, Blecha F: Porcine liver-expressed antimicrobial peptides, hepcidin and LEAP-2: cloning and induction by bacterial infection. Dev Comp Immunol 2006, 30:357-366

6. Rincker MJ, Clarke SL, Eisenstein RS, Link JE, Hill GM: Effects of iron supplementation on binding activity of iron regulatory proteins and the subsequent effect on growth performance and indices of hematological and mineral status of young pigs. J Anim Sci 2005, 83:2137-2145

7. Blachier F, Vaugelade P, Robert V, Kibangou B, Canonne-Hergaux F Delpal S, Bureau F, Blottière $H$, Bouglé D: Comparative capacities of the pig colon and duodenum for luminal iron absorption. Can J Physiol Pharmacol 2007, 85:85-92

8. Schook L, Beattie C, Beever J, Donovan S, Jamison R, Zuckermann F, Niemi S, Rothschild M, Rutherford M, Smith D: Swine in biomedical research: creating the building blocks of animal models. Anim Biotechnol 2005, 16:183-190

9. Quintero-Gutiérrez AG, González-Rosendo G, Sánchez-Muñoz J Polo-Pozo J, Rodríguez-Jerez JJ: Bioavailability of heme iron in biscuit filling using piglets as an animal model for humans. Int J Biol Sci 2008, 4:58-62

10. Brady PS, Ku PK, Ullrey DE, Miller ER: Evaluation of an amino acidiron chelate hematinic for the baby pig. J Anim Sci 1978, 47: 1135-1140

11. Egeli AK, Framstad T: An evaluation of iron-dextran supplementation in piglets administered by injection on the first, third or fourth day after birth. Res Vet Sci 1999, 66:179-184

12. Mahan DC, Shields RG Jr: Macro- and micromineral composition of pigs from birth to 145 kilograms of body weight. J Anim Sci 1998 , 76:506-512

13. Nemeth E, Ganz T: Regulation of iron metabolism by hepcidin. Annu Rev Nutr 2006, 26:323-342

14. Skrzypek T, Valverde Piedra JL, Skrzypek H, Kazimierczak W, Biernat M, Zabielski R: Gradual disappearance of vacuolated enterocytes in the small intestine of neonatal piglets. J Physiol Pharmacol 2007, 58 Suppl 3:87-95

15. Torrance JD, Bothwell TH: Tissue iron stores, Methods in Hematology, vol 1, Edited by JD Cook. New York, Churchill Livingstone, 1980, $90-115$

16. Leong LM, Tan BH, Ho KK: A specific stain for the detection of nonheme iron proteins in polyacrylamide gels. Anal Biochem 1992 207:317-320

17. Simpson DAC, Feeney S, Boyle C, Stitt AW: Retinal VEGF mRNA measured by SYBR green I fluorescence: a versatile approach to quantitative PCR. Mol Vis 2000, 6:178-183

18. Canonne-Hergaux F, Gruenheid S, Ponka P, Gros P: Cellular and subcellular localization of the Nramp2 iron transporter in the intestinal brush border and regulation by dietary iron. Blood 1999, 93:4406-4417

19. Siomek A, Gackowski D, Rozalski R, Dziaman T, Szpila A, Guz J, Olinski R: Higher leukocyte 8-oxo-7,8-dihydro-2'-deoxyguanosine and lower plasma ascorbate in aging humans? Antioxid Redox Signal 2007, 9:143-150

20. Speina E, Zielińska M, Barbin A, Gackowski D, Kowalewski J, Grąziewicz M-A, Siedlecki JA, Oliński R, Tudek B: Decreased repair activities of $1, \mathrm{~N}^{6}$-ethenoadenine and $3, \mathrm{~N}^{4}$-ethenocytosine in lung adenocarcinoma patients Cancer Res 2003, 63:4351-4357

21. Pigeon C, Ilyin G, Courselaud B, Leroyer P, Turlin B, Brissot P, Loréal $O$ : A new mouse liver-specific gene, encoding a protein homologous to human antimicrobial peptide hepcidin, is overexpressed during iron overload. J Biol Chem 2001, 276:7811-7819

22. Lin L, Valore EV, Nemeth E, Goodnough JB, Gabayan V, Ganz T: Iron transferrin regulates hepcidin synthesis in primary hepatocyte culture through hemojuvelin and BMP2/4. Blood 2007, 110:2182-2189

23. McKie AT, Marciani P, Rolfs A, Brennan K, Wehr K, Barrow D, Miret S, 
Bomford A, Peters TJ, Farzaneh F, Hediger MA, Hentze MW, Simpson RJ: A novel duodenal iron-regulated transporter. IREG1, implicated in the basolateral transfer of iron to the circulation. Mol Cell 2000, 5:299-309

24. Gackowski D, Kruszewski M, Bartlomiejczyk T, Jawien A, Ciecierski M, Olinski R: The level of 8-oxo-7,8-dihydro-2'-deoxyguanosine is positively correlated with the size of the labile iron pool in human lymphocytes. J Biol Inorg Chem 2002, 7:548-550

25. Cooke MS, Olinski R, Evans MD: Does measurement of oxidative damage to DNA have clinical significance? Clin Chim Acta 2006, 365:30-49

26. Tudek B: Base excision repair modulation as a risk factor for human cancers. Mol Aspects Med 2007, 28:258-275

27. Venn JAJ, McCance RA, Widdowson EM: Iron metabolism in piglet anemia. J Comp Pat 1947, 57:314-325

28. Egeli AK, Framstad T: Evaluation of the efficacy of perorally administered glutamic acid-chelated iron and iron-dextran injected subcutaneously in Duroc and Norwegian Landrace piglets. Zentralbl Veterinarmed A 1998, 45:53-61

29. Szabo P, Bilkei G: Iron deficiency in outdoor pig production. J Vet Med A Physiol Pathol Clin Med 2002, 49:390-391

30. Baintner K: Vacuolation in the young. Biology of the Intestine in Growing Animals. Edited by R Zabielski, PC Gregory, and B Weström. Amsterdam, Elsevier 2002, pp 55-110

31. Leong WI, Bowlus CL, Tallkvist J, Lönnerdal B: Iron supplementation during infancy - effects on expression of iron transporters, iron absorption, and iron utilization in rat pups. Am J Clin Nutr 2003, 78:1203-1211

32. Lopez V, Suzuki YA, Lönnerdal B: Ontogenic changes in lactoferrin receptor and DMT1 in mouse small intestine: implications for iron absorption during early life. Biochem Cell Biol 2006, 84:337-344

33. De Domenico I, Ward DM, Langelier C, Vaughn MB, Nemeth E, Sundquist WI, Ganz T, Musci G, Kaplan J: The molecular mechanism of hepcidin-mediated ferroportin down-regulation. Mol Biol Cell 2007, 18:2569-2578

34. Nicolas G, Chauvet C, Viatte L, Danan JL, Bigard X, Devaux I, Beaumont C, Kahn A, Vaulont S: The gene encoding the iron regulatory peptide hepcidin is regulated by anemia, hypoxia, and inflammation. J Clin Invest 2002, 110:1037-1044

35. Marchini G, Berggren V, Djilali-Merzoug R, Hansson LO: The birth process initiates an acute phase reaction in the fetus-newborn infant. Acta Paediatr 2000, 89:1082-1086

36. Nemeth E, Rivera S, Gabayan V, Keller C, Taudorf S, Pedersen BK, Ganz T: IL-6 mediates hypoferremia of inflammation by inducing the synthesis of the iron regulatory hormone hepcidin. J Clin Invest 2004, 113:1271-1276

37. Lönnerdal B, Kelleher SL: Iron metabolism in infants and children. Food Nutr Bull 2007, 28:S491-S499

38. Bondi A, Valentino P, Daraio F, Porporato P, Gramaglia E, Carturan S, Gottardi E, Camaschella C, Roetto A: Hepatic expression of hemochromatosis genes in two mouse strains after phlebotomy and iron overload. Haematologica 2005, 90:1161-1167

39. Chaston T, Chung B, Mascarenhas M, Marks J, Patel B, Srai SK Sharp P: Evidence for differential effects of hepcidin in macrophages and intestinal epithelial cells. Gut 2008, 57:374-382

40. Ganz T, Olbina G, Girelli D, Nemeth E, Westerman M: Immunoassay for human serum hepcidin. Blood 2008, 112:4292-4297
41. Busbridge M, Griffiths C, Ashby D, Gale D, Jayantha A, Sanwaiya A, Chapman RS: Development of a novel immunoassay for the iron regulatory peptide hepcidin. Br J Biomed Sci 2009, 66:150-157

42. Koliaraki V, Marinou M, Vassilakopoulos TP, Vavourakis E, Tsochatzis E, Pangalis GA, Papatheodoridis G, Stamoulakatou A, Swinkels DW, Papanikolaou G, Mamalaki A: A novel immunological assay for hepcidin quantification in human serum. PLoS One 2009, 4:e4581

43. Kroot JJ, Kemna EH, Bansal SS, Busbridge M, Campostrini N, Girelli D, Hider RC, Koliaraki V, Mamalaki A, Olbina G, Tomosugi N, Tselepis C, Ward DG, Ganz T, Hendriks JC, Swinkels DW: Results of the first international round robin for the quantification of urinary and plasma hepcidin assays: need for standardization. Haematologica 2009, 94:1748-1752

44. Ueberschär S: Sudden death in suckling piglets following administration of iron-dextran-preparation. Dtsch Tierarztl Wochenschr 1966 73:145-150

45. Anderson GJ, Darshan D, Wilkins SJ, Frazer DM: Regulation of systemic iron homeostasis: how the body responds to changes in iron demand. Biometals 2007, 20:665-674

46. Geisser P, Baer M, Schaub E: Structure/histotoxicity relationship of parenteral iron preparations. Arzneimittelforschung 1992, 42:1439-1452

47. Richter GW: Studies of iron overload. Lysosomal proteolysis of rat liver ferritin. Pathol Res Pract 1986, 181:159-167

48. Hultcrantz R, Glaumann $\mathrm{H}$ : Studies on the rat liver following iron overload: biochemical studies after iron mobilization. Lab Invest 1982, 46:383-392

49. Olinski R, Gackowski D, Rozalski R, Foksinski M, Bialkowki K: Oxidative DNA damage in cancer patients: a cause or a consequence of the disease development? Mutat Res 2003, 531:177-190

50. Russo MT, De Luca G, Degan P, Parlanti E, Dogliotti E, Barnes DE, Lindahl T, Yang H, Miller JH, Bignami M: Accumulation of the oxidative base lesion 8-hydroxyguanine in DNA of tumor-prone mice defective in both the Myh and Ogg1 DNA glycosylases. Cancer Res 2004, 64:4411-4414

51. Pines A, Perrone L, Bivi N, Romanello M, Damante G, Gulisano M, Kelley MR, Quadrifoglio F, Tell G: Activation of APE1/Ref-1 is dependent on reactive oxygen species generated after purinergic receptor stimulation by ATP. Nucleic Acids Res 2005, 33:4379-4394

52. Bouziane M, Miao F, Bates SE, Somsouk L, Sang BC, Denissenko M, O'Connor TR: Promoter structure and cell cycle dependent expression of the human methylpurine-DNA glycosylase gene. Mutat Res 2000, 461:15-29

53. Delker D, Hatch G, Allen J, Crissman B, George M, Geter D, Kilburn S, Moore T, Nelson G, Roop B, Slade R, Swank A, Ward W, DeAngelo A: Molecular biomarkers of oxidative stress associated with bromate carcinogenicity. Toxicology 2006, 221:158-165

54. Galleano M, Simontacchi M, Puntarulo S: Nitric oxide and iron: effect of iron overload on nitric oxide production in endotoxemia. Mol Aspects Med 2004, 25:141-154

55. Lipinski P, Starzynski RR, Drapier JC, Bouton C, Bartlomiejczyk T, Sochanowicz B, Smuda E, Gajkowska A, Kruszewski M: Induction of iron regulatory protein 1 RNA binding activity by nitric oxide is associated with a concomitant increase in the labile iron pool: implications for DNA damage. Biochem Biophys Res Commun 2005, 327:349-355 